\title{
Quality of life in patients receiving home parenteral nutrition
}

\author{
P B Jeppesen, E Langholz, P B Mortensen
}

\begin{abstract}
BackgroundlAims-Quality of life is an important determinant of the effectiveness of health technologies, but it has rarely been assessed in patients receiving home parenteral nutrition (HPN).

Patients/Methods-The non-disease specific sickness impact profile (SIP) and the disease specific inflammatory bowel disease questionnaire (IBDQ) were used on a cohort of 49 patients receiving $\mathrm{HPN}$, and the results compared with those for 36 non-HPN patients with either anatomical $(<200 \mathrm{~cm})$ or functional (faecal energy excretion $>2.0 \mathrm{MJ} /$ day $(\sim 488 \mathrm{kcal} /$ day $))$ short bowel.

Results-In the HPN patients the SIP scores were worse (higher) overall (17 $(13) \% v 8(9) \%)$ and with regard to physical $(13(15) \% v 5(8) \%)$ and psychosocial (14 (12)\% $v 9(11) \%)$ dimensions and independent categories $(20(12) \%$ v 9 (8)\%) compared with the non-HPN patients (means (SD); all p<0.001). The IBDQ scores were worse (lower) in the HPN patients overall (5.0 (4.3-5.7) $v 5.6$ (4.8$6.2)$ ) and with regard to systemic symptoms (3.8 (2.8-5.4) $v 5.2(3.9-5.9))$ and emotional (5.3 (4.4-6.2) $v 5.8$ (5.4-6.4)) and social $(4.3(3.4-5.5)$ v $4.8(4.5-5.8))$ function (median (25-75\%); all p<0.05), but only tended to be worse with regard to bowel symptoms (5.2 (4.8-6.1) $v 5.7$ (4.9$6.4), p=0.08)$. HPN also reduced quality of life in patients with a stoma, whereas a stoma did not reduce quality of life among the non-HPN patients. Female HPN patients and HPN patients older than 45 scored worse.

Conclusion-Quality of life is reduced in patients on HPN compared with those with anatomical or functional short bowel not receiving HPN, and compares with that reported for patients with chronic renal failure treated by dialysis.

(Gut 1999;44:844-852)
\end{abstract}

Keywords: parenteral nutrition; quality of life; sickness impact profile; inflammatory bowel disease

Parenteral nutrition is a lifesaving procedure in patients who have intestinal failure defined as inadequate intestinal function for absorption of nutrients and electrolytes. ${ }^{1}$ While in hospital, the patients who can be maintained on parenteral nutrition are educated in the aseptic infusion of nutrients and electrolytes, thereby avoiding the metabolic disturbances and malnutrition seen as a consequence of intestinal failure. When qualified in this complex procedure, the patients are discharged for home parenteral nutrition (HPN). Intestinal transplantation on the other hand is the ultimate lifesaving option when complications impede parenteral support, such as progressive liver failure, serial septic episodes, and venous inaccessibility, most frequently seen in the paediatric population. ${ }^{23}$

Moving the parenteral support from the hospital to the home results in a significant gain in quality of life, ${ }^{4}$ and as intestinal adaptation occurs, some patients may even be weaned off parenteral supplements. Others, however, experience irreversible intestinal failure and face life-long complex technological nutritional support, which inevitably has an impact on their quality of life. HPN is a time consuming intrusive procedure, and HPN patients with intestinal failure are often troubled by the inconvenience of high intestinal output, presence of a stoma, fear of incontinence, altered body image, etc. These factors may impose severe restrictions on daily life with regard to social and leisure activities and emotional function, and the presence of malnutrition and dehydration in spite of HPN therapy may affect physical activity. In these patients the quality control of medical care must be focused towards proper control of the symptoms and complications of intestinal failure and treatment with HPN, aimed at full rehabilitation of the HPN patient.

As the results of intestinal transplantation will probably improve in the coming years, this procedure may become an alternative to HPN on the lines of renal transplantation versus dialysis, not only on vital indications, but also with the aim of improving quality of life in these patients.

In order to understand the experience of chronic illness and to describe behavioural dysfunction and problems related to HPN treatment, comparisons were made, using validated quality of life measurement techniques, between a population of patients receiving HPN monitored at the intestinal failure unit in Copenhagen in July 1997 and a group of nonHPN patients with known severe malabsorption, who managed without parenteral supplements.

Materials and methods

QUESTIONNAIRES

The study was based on two validated quality of life questionnaires: the sickness impact

Abbreviations used in this paper: $\mathrm{HPN}$, home parenteral nutrition; IBDQ, inflammatory bowel disease questionnaire; SIP, sickness impact profile.
Rigshospitalet, Blegdamsvej

Denmark.

Accepted for publication 6 January 1999 
profile $(\mathrm{SIP})^{5}$ and the inflammatory bowel disease questionnaire (IBDQ) ${ }^{6}$

The SIP is a non-disease specific behaviour based measure of sickness related dysfunction designed to cover patient perception of performance in areas of activity in everyday life. It contains 136 items in two main dimensions (physical (ambulation and mobility, body care and movement) and psychosocial (social interaction, alertness and emotional behaviour, communication)) and five independent categories (sleep and rest, eating, work, house keeping, recreation and pastimes). It is designed to be broadly applicable across types and severities of illness and across demographic and cultural subgroups. It has been used to collect and evaluate sickness related behavioural dysfunction in various diseases ${ }^{78}$ and was chosen for this study to provide a measure of the non-disease specific function of the two groups of patients. Patients were asked to endorse or check those statements that accorded with their present situation. No positive answers was equivalent to no behavioural dysfunction. The SIP percentage scores of the dimensions and categories were obtained by summing the number of positive statements to the items in each dimension and category, dividing that sum by the total sum of the possible values, and multiplying the quotient by 100. Zero per cent indicates the best possible function (absence of dysfunction), whereas $100 \%$ indicates presence of all possible dysfunctional behaviour.

At the end of the SIP questionnaire, patients were asked to mark their overall quality of life on a $9 \mathrm{~cm}$ visual analogue scale. At the left at 0 $\mathrm{cm}$ a miserable quality of life was indicated, whereas an ideal quality of life was indicated at $9 \mathrm{~cm}$ at the right end of the scale.

The IBDQ was developed to measure subjective health status for patients with inflammatory bowel disease. The 32 item questionnaire examines four aspects of patients' lives: symptoms directly related to the primary bowel disturbance (10 questions), systemic symptoms (five questions), emotional (12 questions), and social function (five questions). This questionnaire is disease specific and was chosen to focus on bowel related symptoms and their impact on quality of life. The response options for each question were framed as a seven point scale on which 7 represented best function and 1 represented worst function. The score of each aspect has been given as a median on the seven point scale.

EXPERIMENTAL DESIGN AND PATIENTS

In June 1997 the two questionnaires were mailed to the total cohort of 57 patients (corresponding to $75 \%$ of patients receiving HPN in Denmark) followed at the intestinal failure unit in Copenhagen receiving HPN because of intestinal failure secondary to benign disease, and to 45 non-HPN patients, who had an anatomical and/or functional short bowel defined as $<200 \mathrm{~cm}$ of remnant small bowel (26 patients) or a daily faecal energy loss measured by bomb calorimetry exceeding 2.0 $\mathrm{MJ} /$ day ( 488 $\mathrm{kcal} /$ day) during their last admission (37 patients). Written reminders were sent to non-responders after two months and the study was closed for inclusion at three months.

The population of patients receiving HPN in Denmark and the standardised care of these patients has been described in a recent study. ${ }^{9}$ None of the patients had a history of an underlying psychiatric disorder. In only one HPN patient was the impairment of quality of life evidently secondary to the underlying disease and not necessarily related directly to HPN therapy. This patient suffered from CharcotMarie-Tooth syndrome, had intestinal dysmotility, and was partly immobilised as a result of the disease.

The HPN patients and their relatives were trained by a special team. An instruction manual was handed out, and the patients were discharged from hospital when they were able to carry out the procedures. Thus the HPN consumers were taught to be totally independent of nursing involvement with routine infusion. Home care companies delivered the HPN products, and even supplied them if the patients were away from their home town. Single-lumen catheters, inserted through the subclavian, jugular, or femoral vein and advanced to the vena caval-right atrial junction, were used. Administration was generally at night, but six patients with large stomal volumes had additional infusions of saline during the day. The recommended infusion time of standard 3 litre HPN bags was 10 hours. Infusion was by gravity in all patients.

The patients had 24 hour access to the intestinal failure unit in Copenhagen for emergencies. Nursing support at home was instituted for seven patients. All patients in this study were monitored in our outpatient clinic at intervals of about 6-12 weeks. During these visits patients were clinically assessed, weighed, and routine blood tests taken. At intervals of about one year, intestinal function was assessed using balance techniques that measured diet and faecal weight and energy content by bomb calorimetry. In the HPN patients the parenteral energy and electrolyte supplements were adjusted on a clinical basis to maintain normal body weight, hydration, diuresis, and levels of plasma albumin and electrolytes. This information was obtained from medical records, and remnant intestinal length was obtained from surgical records. The length of the colon was expressed in terms of percentage of the usual length by the method of Cummings et $a l .{ }^{10}$ Basal energy expenditure was calculated by Harris-Benedict equations using actual body weights. ${ }^{11}$

ETHICS

All procedures were performed in accordance with the ethical standards of the Helsinki Declaration of 1975, as revised in 1983. Patients gave their informed consent.

STATISTICAL ANALYSIS

A non-parametric Mann-Whitney rank sum test was used for the comparison of patient characteristics of the two study groups, the 
Table 1 Demographics of patients on home parenteral nutrition (HPN) and those not

\begin{tabular}{llll}
\hline & $H P N(n=49)$ & Non-HPN $(n=36)$ & $p$ Value \\
\hline Sex (female/male) & $31 / 18$ & $20 / 16$ & $0.62 \dagger$ \\
Diagnosis (CD/MD+OP/dysmotility) & $(31 / 8 / 10)$ & $(30 / 6 / 0)$ & $0.01 \dagger$ \\
Age (years) & $45.4(37.7-56.9)$ & $50.0(44.1-60.4)$ & $0.16^{\star}$ \\
Height $(\mathrm{cm})$ & $167(163-174)$ & $170(162-175)$ & $0.47^{\star}$ \\
Weight $(\mathrm{kg})$ & $57.4(51.3-64.9)$ & $63.2(56.1-69.3)$ & $0.03^{\star}$ \\
Body mass index $\left(\mathrm{kg} / \mathrm{m}^{2}\right)$ & $20.8(18.9-22.8)$ & $22.2(20.0-24.0)$ & $0.046^{\star}$ \\
Remnant small bowel $(\mathrm{cm})$ & $140(74-233)$ & $200(148-246)$ & $0.03^{\star}$ \\
Remnant colon $(\%)$ & $0(0-64)$ & $29(0-86)$ & $0.21^{\star}$ \\
Patients with a stoma (n) & 38 & 17 & $0.008 \dagger$ \\
Diet energy intake & & & \\
$\quad(\mathrm{MJ} /$ day) & $8.12(6.30-10.17)$ & $11.49(9.13-13.56)$ & $<0.001^{\star}$ \\
$\quad$ Encal/day) & $1941(1505-2429)$ & $2745(2182-3238)$ & \\
Faecal weight $(\mathrm{kg} /$ day) & $72(50-94)$ & $127(113-150)$ & $<0.001^{\star}$ \\
\hline Resuls are expsorption/BEE $\%$ & $1.87(0.95-2.80)$ & $1.25(0.67-1.71)$ & $0.03^{\star}$ \\
\hline
\end{tabular}

Results are expressed as median (25-75\%). ${ }^{\star}$ The Mann-Whitney rank sum test or the $+\chi^{2}$ test was used for comparison between groups. $\mathrm{CD}$, Crohn's disease; $\mathrm{MD}+\mathrm{OP}$, patients with intestinal resections because of mesenteric vascular disease or complications of intra-abdominal surgery. BEE, basal energy expenditure calculated by the Harris-Benedict equations using actual body weights. $^{11}$

scores on the visual analogue scale in the SIP questionnaires, and the medians between groups in the IBDQ questionnaires. The $\chi^{2}$ or Fisher exact test was used for comparison of the frequencies of confirmatory answers in the SIP questionnaire. The statistical software used was SigmaStat for Windows Version 2.0 (copyright 1992-1995; Jandel Corporation, Erkrath, Germany). $\mathrm{p}<0.05$ was considered to indicate statistical significance.

\section{Results}

PATIENT DEMOGRAPHICS

Forty nine $(86 \%)$ of the HPN patients and 36 $(80 \%)$ of the non-HPN patients returned completed questionnaires; table 1 gives the demographics of these patients. The median duration of HPN treatment was 5.0 (range $0.2-27.8$ ) years. The HPN patients were given a median of 2.23 (range 0.2-5.5) litres of parenteral fluid per day and 3.97 (range 0.0-10.5) MJ/day ( 948 (range 0-2508) kcal/ day) corresponding to a median of $73 \%$ of their basal energy expenditure. The HPN was infused for a median of seven (range four to seven) nights on a cyclic nocturnal basis, but six patients had saline supplements during daytime. The two groups did not differ significantly with regard to sex ratio or age. Significantly more of the patients receiving HPN had a dysmotility disorder. Body mass index was lower in the HPN patients because of a lower body weight compared with the non-HPN patients. The remnant small bowel was significantly shorter and the presence of a stoma more predominant in the HPN patients compared with the non-HPN patients. In spite of a lower dietary energy intake, the HPN patients had a higher faecal weight than the non-HPN patients. The energy absorption in relation to the basal energy expenditure was $72 \%$ and $127 \%$ in the two groups respectively $(\mathrm{p}<0.001)$.

\section{SIP SCORES}

Figure 1 gives a comparison of the overall SIP scores, dimensions, and categories between the HPN and non-HPN patients. A score of $0 \%$ indicates the best possible function (absence of dysfunction), whereas $100 \%$ indicates presence of all possible dysfunctional behaviour. The patients receiving HPN scored worse (higher scores) in all areas of activity. All 136 questions were individually compared in order to identify differences among the HPN and the non-HPN patients.

\section{Physical dimension}

The responses to questions on ambulation and mobility showed that the HPN patients used public transport less frequently (41\%) than the non-HPN patients $(11 \%)(p=0.006)$. The HPN patients reported that they spent more

\begin{tabular}{|c|c|c|c|c|}
\hline & & & ore & $\mathrm{p}$ value \\
\hline Dimension & Category & HPN & non-HPN & \\
\hline & Ambulation and mobility (22) & $17(19) \%$ & $6(13) \%$ & $<0.001$ \\
\hline Pnysical & Body care and movement (23) & $10(15) \%$ & $4(7) \%$ & $<0.001$ \\
\hline & Total physical (45) & $13(15) \%$ & $5(8) \%$ & $<0.001$ \\
\hline & Social interaction (20) & $18(13) \%$ & $11(15) \%$ & $<0.001$ \\
\hline SIP — Psychosocial & Alertness and emotional behaviour (19) & $18(20) \%$ & $10(15) \%$ & 0.007 \\
\hline & Communication (9) & $3(11) \%$ & $1(4) \%$ & 0.010 \\
\hline & Total psychosocial (48) & $14(12) \%$ & $9(11) \%$ & $<0.001$ \\
\hline 1 & Sleep and rest (7) & $21(16) \%$ & $12(14) \%$ & 0.010 \\
\hline Independent & Eating (9) & $20(12) \%$ & $2(5) \%$ & $<0.001$ \\
\hline categories & Work (9) & $86(35) \%$ & $56(50) \%$ & 0.004 \\
\hline & Home management (10) & $25(22) \%$ & $10(14) \%$ & $<0.001$ \\
\hline & Recreation and pastimes (8) & $32(27) \%$ & $15(20) \%$ & $<0.001$ \\
\hline & Total independent categories (41) & $20(12) \%$ & $9(8) \%$ & $<0.001$ \\
\hline Overall (136) & & $17(13) \%$ & $8(9) \%$ & $<0.001$ \\
\hline VAS score $(0-9$ & $\mathrm{n}, 9 \mathrm{~cm}$ best) & $4.9(2.4) \mathrm{cm}$ & $6.8(2.2) \mathrm{cm}$ & 0.008 \\
\hline
\end{tabular}

Figure 1 Comparison of SIP scores between patients receiving home parenteral nutrition (HPN) and those who did not. Results are expressed as mean (SD). Frequencies of confirmatory answers in the SIP questionnaire were compared between groups using the $\chi^{2}$ test or alternatively Fisher's exact test. The VAS scores were compared using a Mann-Whitney rank sum test. The values in parentheses in the category column give the numbers of items in each category. Zero per cent indicates the best possible function (absence of dysfunction), whereas $100 \%$ indicates presence of all possible dysfunctional behaviour. 
Table 2 Inflammatory bowel disease questionnaire (IBDQ) scores for patients on home parenteral nutrition (HPN) and those not

\begin{tabular}{|c|c|c|c|}
\hline & $H P N$ & non-HPN & $p$ Value \\
\hline \multicolumn{4}{|l|}{ Bowel symptoms } \\
\hline Bowel movement frequency & $7.0(4.0-7.0)$ & $7.0(4.0-7.0)$ & 0.57 \\
\hline Loose bowel movements & $1.0(1.0-6.2)$ & $3.0(1.0-4.0)$ & 0.02 \\
\hline Cramps in abdomen & $7.0(3.0-7.0)$ & $7.0(6.0-7.0)$ & 0.19 \\
\hline Pain in abdomen & $4.0(2.0-7.0)$ & $7.0(5.0-7.0)$ & 0.01 \\
\hline Passing gas & $7.0(5.0-7.0)$ & $7.0(3.0-7.0)$ & 0.16 \\
\hline Abdominal boating & $7.0(3.3-7.0)$ & $7.0(3.3-7.0)$ & 0.99 \\
\hline Rectal bleeding & $7.0(7.0-7.0)$ & $7.0(7.0-7.0)$ & 0.47 \\
\hline Bathroom though bowel empty & $7.0(7.0-7.0)$ & $7.0(7.0-7.0)$ & 0.59 \\
\hline Accidental soiling of underpants & $7.0(4.0-7.0)$ & $7.0(4.0-7.0)$ & 0.85 \\
\hline Feeling sick to the stomach & $5.0(4.0-7.0)$ & $7.0(6.5-7.0)$ & 0.02 \\
\hline Total & $5.2(4.8-6.1)$ & $5.7(4.9-6.4)$ & 0.08 \\
\hline \multicolumn{4}{|l|}{ Systemic symptoms } \\
\hline Feeling of fatigue/tiredness & $4.0(2.0-5.0)$ & $4.0(3.0-7.0)$ & 0.047 \\
\hline Feeling of energy & $3.0(2.0-4.0)$ & $3.3(3.0-4.0)$ & 0.056 \\
\hline General unwell feeling & $4.0(3.0-6.8)$ & $4.5(3.0-7.0)$ & 0.20 \\
\hline Poor sleeping or frequent wakening & $3.0(1.0-7.0)$ & $4.0(3.0-7.0)$ & 0.02 \\
\hline Problems to maintain weight & $7.0(6.0-7.0)$ & $7.0(7.0-7.0)$ & 0.42 \\
\hline Total & $3.8(2.8-5.4)$ & $5.2(3.9-5.9)$ & 0.008 \\
\hline \multicolumn{4}{|l|}{ Emotional function } \\
\hline Frustrated, impatient, restless & $5.5(3.0-7.0)$ & $7.0(4.0-7.0)$ & 0.11 \\
\hline Worries of new surgery & $7.0(5.0-7.0)$ & $7.0(4.5-7.0)$ & 0.45 \\
\hline Fear of not finding washroom & $7.0(4.8-7.0)$ & $7.0(4.0-7.0)$ & 0.83 \\
\hline Feeling depressed or discouraged & $5.0(3.0-7.0)$ & $7.0(4.0-7.0)$ & 0.11 \\
\hline Worries of cancer or illness & $7.0(6.0-7.0)$ & $7.0(7.0-7.0)$ & 0.26 \\
\hline Relaxed and free of tension & $3.0(1.0-5.8)$ & $4.0(3.0-6.0)$ & 0.057 \\
\hline Embarrassment due to bowel disease & $7.0(7.0-7.0)$ & $7.0(4.0-7.0)$ & 0.13 \\
\hline Feeling tearful or upset & $6.0(4.0-7.0)$ & $7.0(6.0-7.0)$ & 0.066 \\
\hline Anger due to bowel disease & $6.0(3.0-7.0)$ & $7.0(6.3-7.0)$ & 0.01 \\
\hline Irritability & $5.0(4.0-7.0)$ & $5.5(4.0-7.0)$ & 0.44 \\
\hline Lack of understanding from others & $7.0(4.0-7.0)$ & $7.0(6.8-7.0)$ & 0.41 \\
\hline Feeling satisfied, happy, pleased & $4.0(3.0-5.0)$ & $5.0(4.0-5.0)$ & 0.046 \\
\hline Total & $5.3(4.4-6.2)$ & $5.8(5.4-6.4)$ & 0.04 \\
\hline \multicolumn{4}{|l|}{ Social function } \\
\hline Work & $1.0(1.0-1.0)$ & $1.0(1.0-7.0)$ & 0.15 \\
\hline Delay/cancel social arrangements & $7.0(4.5-7.0)$ & $7.0(7.0-7.0)$ & 0.15 \\
\hline Difficulties in leisure/sports activities & $5.0(1.0-7.0)$ & $7.0(2.5-7.0)$ & 0.12 \\
\hline Avoiding events with no washroom close at hand & $7.0(7.0-7.0)$ & $7.0(7.0-7.0)$ & 0.42 \\
\hline Limitations in sexual activity & $1.0(1.0-6.0)$ & $1.0(1.0-6.0)$ & 1.00 \\
\hline Total & $4.3(3.4-5.5)$ & $4.8(4.5-5.8)$ & 0.03 \\
\hline Overall & $5.0(4.3-5.7)$ & $5.6(4.8-6.2)$ & 0.03 \\
\hline
\end{tabular}

The scores of each aspect are given as median (25-75\%) on a seven point scale. 7 represents best function and 1 represents worst function. The Mann-Whitney rank sum test was used for comparison between groups.

time at home than the non-HPN patients (43\% $v 19 \%, \mathrm{p}=0.04)$, and social events and visits were shorter $(35 \% v 8 \%, \mathrm{p}=0.01)$. In response to questions on body care and movement, $17 \%$ of the HPN patients reported needing help for difficult movements - for example, getting into a car and getting out of the bath-compared with $0 \%$ in the non-HPN group ( $\mathrm{p}=0.04)$, and the HPN patients had more difficulty in maintaining their balance $(14 \% v 0 \%, \mathrm{p}=0.04)$.

\section{Psychosocial dimension}

The HPN patients experienced a large impact on their psychosocial activities. As mentioned above, their social interaction was affected because of problems with mobility. The responses to questions on social interaction showed that the HPN patients less frequently paid social visits to others $(49 \% \vee 17 \%, \mathrm{p}=$ $0.004)$, participated less in social arrangements (41\% v 17\%, p = 0.03), and were more often alone $(29 \% v 5 \%, \mathrm{p}=0.02)$ than the non-HPN patients. Concerning alertness and emotional behaviour, the HPN patients in general scored worse (higher score) on questions about emotional stability and self confidence. Some $18 \%$ of the HPN patients felt that they were a nuisance to others compared with $3 \%$ of the non-HPN patients $(\mathrm{p}=0.04)$. However, when asked about their prospects, the answers from the HPN patients were not more futile than those from the non-HPN patients. Some $43 \%$ of the HPN patients reported to have reduced sexual activity, but this did not differ from the non-HPN patients $(42 \%, \mathrm{p}=0.91)$. No significant differences for individual questions on communication were found between the two groups.

\section{Independent categories}

The HPN patients in general had greater sickness related dysfunction with regard to sleep and rest, but none of the differences in the answers to individual questions between the two groups reached statistical significance. For the questions on eating, $41 \%$ of the HPN patients reported having a reduced appetite compared with only $6 \%$ of the non-HPN patients $(p<0.001)$. Only $14 \%$ of the HPN patients were in full time work compared with $44 \%$ of the non-HPN patients ( $p=0.004)$. In questions about home management, $59 \%$ of the HPN patients reported carrying out less of the housework compared with $33 \%$ of the nonHPN patients $(\mathrm{p}=0.03)$ and significantly less did the shopping $(84 \% v 100 \%, \mathrm{p}=0.02)$, cleaning $(76 \%$ v $94 \%, \mathrm{p}=0.04)$, and heavy, demanding work at home $(47 \%$ v $86 \%$, $\mathrm{p}<0.001)$. In questions on recreation and pastimes, the HPN patients spent less time out enjoying themselves $(45 \% v 19 \%, \mathrm{p}=0.03)$ and socialised less $(43 \%$ v 11\%, $\mathrm{p}=0.003)$. They also did less physical training and exercise than the non-HPN patients $(41 \% v$ $17 \%, \mathrm{p}=0.03)$.

On the $9 \mathrm{~cm}$ visual analogue scale measuring the overall feeling of quality of life, the HPN patients had a lower score (median $(25-75 \%)$ $4.9(3.0-7.2) \mathrm{cm})$ than the non-HPN patients $($ median $(25-75 \%) 6.8(4.8-8.2) \mathrm{cm})(\mathrm{p}=$ 0.008).

\section{IBDQ SCORES}

Table 2 gives a comparison of the IBDQ scores between the HPN and non-HPN patients. The response options for each question were framed as a seven point scale on which 7 represented best function and 1 represented worst function. Not only regarding the overall scores, but also in areas of systemic symptoms and emotional and social function, the HPN patients scored worse (lower score) than the non-HPN patients. Significance was not reached for overall bowel symptoms (5.2 $v 5.7$, $\mathrm{p}=0.08)$.

Table 2 also gives responses to the individual questions in the IBDQ. The HPN patients reported more episodes of loose bowel movements and abdominal pain than the non-HPN patients, and they had more nausea and vomiting. Systemically the HPN patients felt more fatigue than the non-HPN patients, and they tended to score worse (lower score) with regard to energy for everyday activities. The HPN patients reported having more problems sleeping than the non-HPN patients. With regard to emotional functions, the HPN patients felt more anger as a result of their bowel problem than the non-HPN patients, and when asked how satisfied, happy, or pleased they were with their personal life, they scored worse (lower score) than the non-HPN patients. None of the 

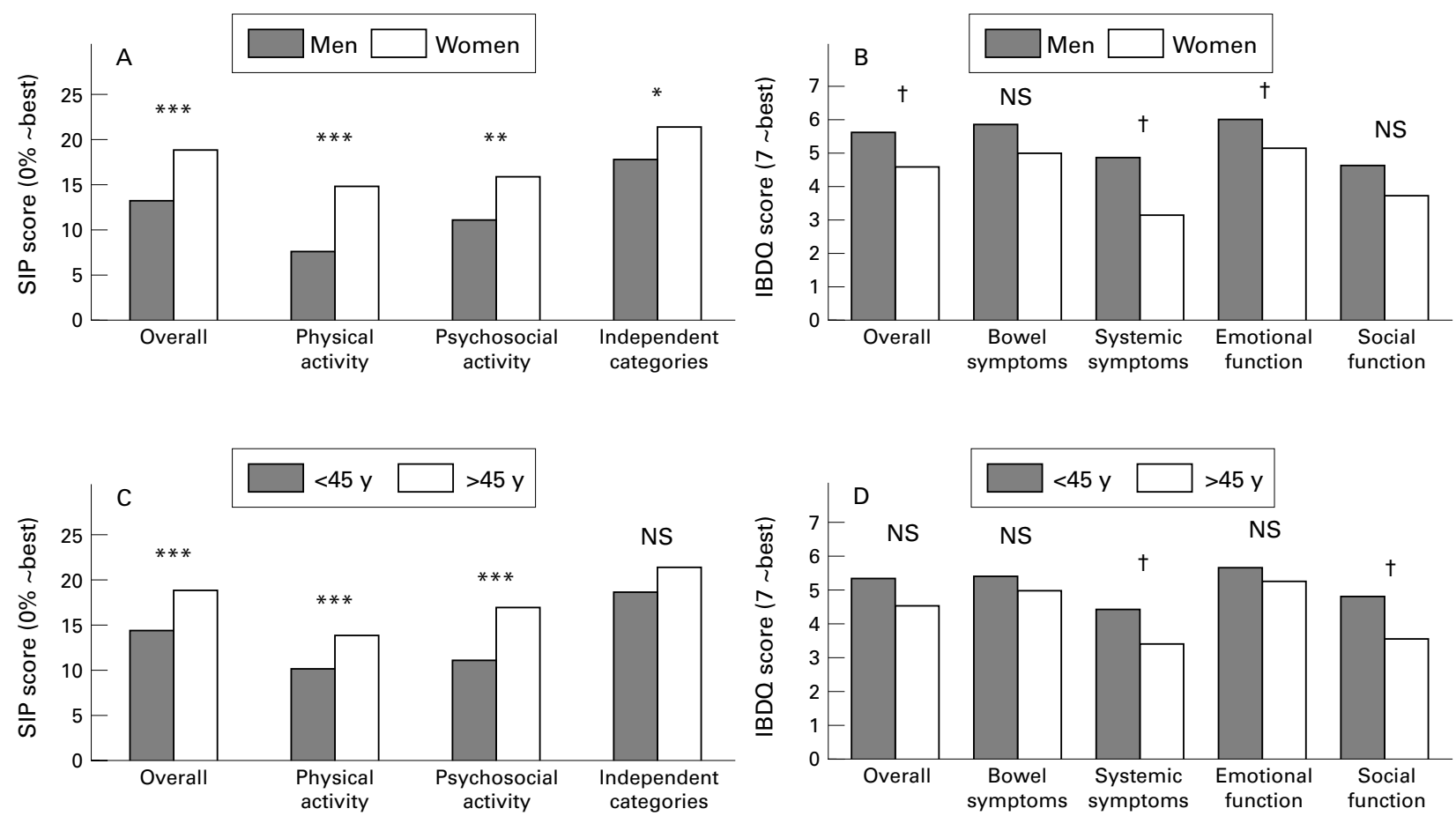

Figure 2 Mean sickness impact profile (SIP) scores and median inflammatory bowel disease questionnaire (IBDQ) scores according to sex (A and B) and age $(C$ and $D)$ in the patients on home parenteral nutrition $(H P N) \cdot \chi^{2}$ or Fisher exact test: ${ }^{\star} p<0.05 ;{ }^{\star *} p<0.01, \star \star \star x<0.001$. Mann-Whitney rank sum test: $t p<0.05$.

differences in responses to individual questions on social function reached statistical significance, but an overall worse score (lower score) was seen in the HPN patients compared with non-HPN patients. No differences were seen between the two groups with regard to limitations in sexual activity. Some $55 \%$ of the HPN patients and $53 \%$ of the non-HPN patients, however, reported that their bowel problem had reduced their sexual activity.

EFFECT OF SEX AND AGE ON SIP AND IBDQ SCORES To evaluate the effect of sex and age on the SIP and IBDQ scores, the HPN patients were divided according to sex and age below and above 45 years. Eighteen patients were men and 31 women, and 23 patients were less than 45 and 26 were more than 45 .

Sex

The median age in the male and female HPN population was 45.4 and 46.1 years respectively $(\mathrm{p}=0.55)$. Figure $2 \mathrm{~A}, \mathrm{~B}$ gives the SIP and IBDQ scores respectively arranged by sex. The female HPN patients scored significantly worse (higher score) overall and for the physical and psychosocial dimensions and the independent categories. An analysis of the physical dimension showed that the female HPN patients had worse SIP scores (higher score) with regard to both ambulation and mobility $(22 \%$ v $10 \%, \mathrm{p}=$ $0.002)$ and body care and movement $(12 \%$ v $6 \%, \mathrm{p}=0.002)$ compared with the male HPN patients. In the psychosocial dimension no significant difference was seen between sexes with regard to social interaction, but the female patients scored worse (higher score) in areas of alertness and emotional behaviour $(22 \% v 11 \%$, $\mathrm{p}=0.009)$ and communication $(5 \% v 1 \%, \mathrm{p}=$ 0.04 ) compared with the male HPN patients.
With respect to the individual categories, no differences were seen between the sexes with regard to sleep and rest, eating, work or recreation and pastimes. The female HPN patients, however, scored significantly worse (higher score) with regard to home management (30\% v $16 \%, \mathrm{p}<0.001)$.

When considering the $9 \mathrm{~cm}$ visual analogue scale for overall feeling of quality of life, the female HPN patients tended to score worse (lower score: median (25-75\%) 4.6 (2.2-7.0) $\mathrm{cm})$ than the male HPN patients $(6.6(4.5-7.4)$ $\mathrm{cm})(\mathrm{p}=0.068)$.

With regard to the IBDQ scores, the female HPN patients scored significantly worse (lower score) overall as well as in the systemic symptoms and emotional function categories. No differences could be shown for bowel symptoms and social function between the sexes (fig 2B). Analysis of the responses to the individual questions on bowel symptoms, however, showed that the female HPN patients scored worse (lower score) in the question on pain in the abdomen (3.0 $v 7.0, \mathrm{p}=0.004)$ and abdominal bloating $(5.0 v 7.0, \mathrm{p}=0.04)$. The female HPN patients scored significantly worse (lower score) in all individual questions on systemic symptoms, except when asked about problems of weight maintenance. Looking at emotional function, the female HPN patients felt more depressed and discouraged $(4.0 v 7.0, \mathrm{p}=0.01)$, more tearful and upset (5.5 $v 7.0, \mathrm{p}=0.003)$, more lack of understanding from others $(7.0 \mathrm{v}$ $7.0, \mathrm{p}=0.02)$, and in general less satisfied, happy, or pleased $(4.0 \approx 5.0, \mathrm{p}=0.01)$.

Age

The male/female ratio in the HPN patients below and above the age of 45 years was $8 / 17$ 

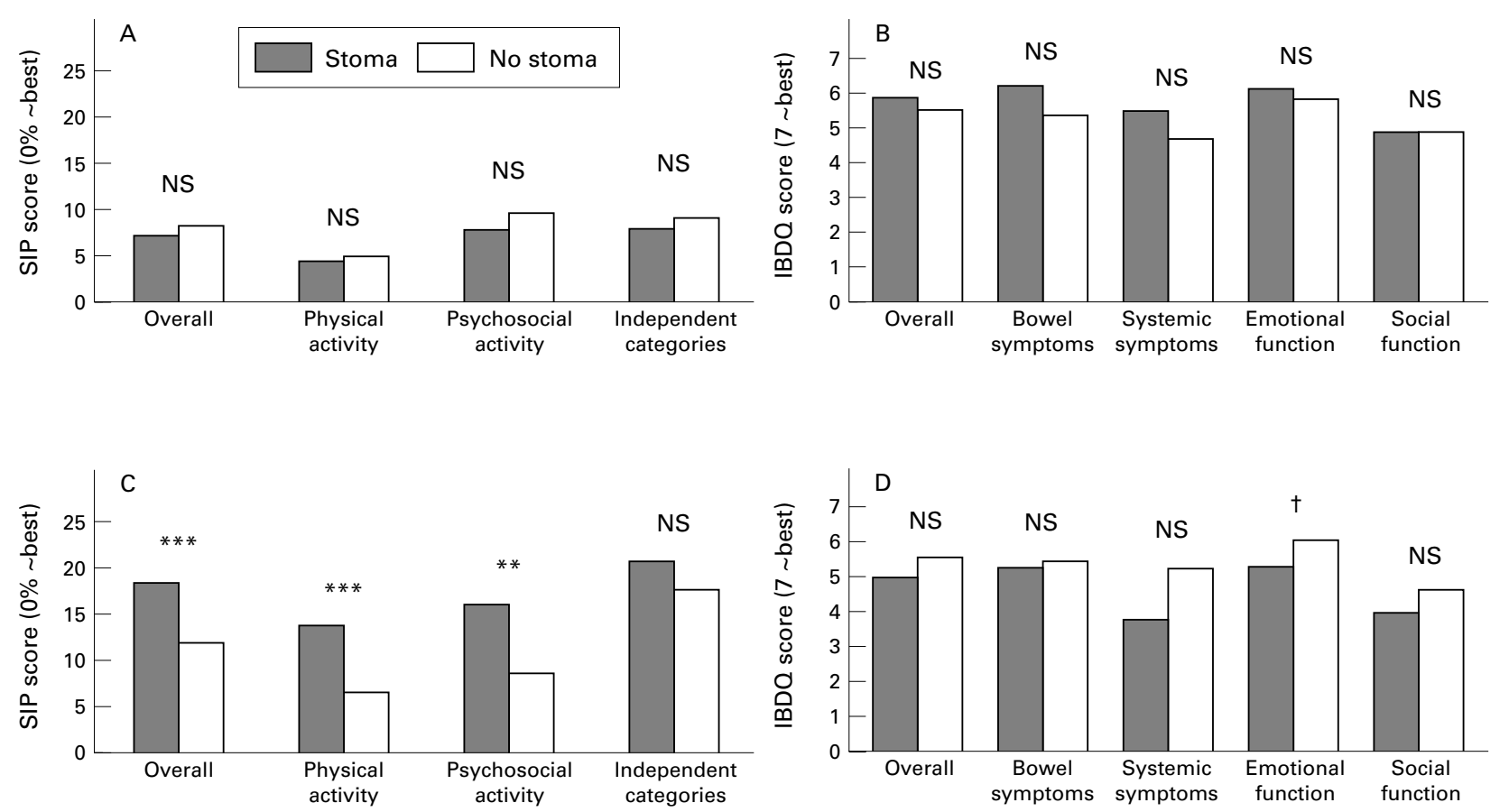

Figure 3 Mean sickness impact profile (SIP) scores and median inflammatory bowel disease questionnaire (IBDQ) scores according to the presence or absence of a stoma in patients not on home parenteral nutrition (HPN) (A and B) and those on HPN (C and D). $\chi^{2}$ or Fisher exact test: ${ }^{\star} p<0.05$; ${ }^{\star *} p<0.01 ;{ }^{\star \star *} p<0.001$. Mann-Whitney rank sum test: $t_{p}<0.01$.

and $10 / 16(\mathrm{p}=0.85)$. Figure $2 \mathrm{C}, \mathrm{D}$ gives a comparison of the SIP and IBDQ scores respectively between the younger and older HPN patients. The patients below the age of 45 years scored significantly better (lower score) on the overall SIP score and the physical and psychosocial dimensions, whereas no differences were encountered for the independent category. An analysis of the categories in the physical dimension showed that body care and movement were better (lower score) in the younger age group (SIP score $8 \% v 12 \%, \mathrm{p}=$ $0.03)$, whereas the differences in responses to questions on ambulation and mobility did not reach statistical significance. In the psychosocial dimension, the SIP score was worse (higher score) with regard to alertness and emotional behaviour in the older HPN patients $(23 \% v$ $12 \%, \mathrm{p}=0.005)$. No differences were encountered for social interaction and communication. In the independent categories no differences were detected with regard to sleep and rest, eating, and recreation and pastimes between younger and older HPN patients, but the younger HPN patients did better (lower score) with regard to work (SIP score $74 \% v$ $96 \%, \mathrm{p}=0.04$ ) and home management (SIP score $17 \%$ v $32 \%, \mathrm{p}<0.001)$ than the older patients.

When considering the $9 \mathrm{~cm}$ visual analogue scale for overall feeling of quality of life, no difference was seen between the younger and older HPN patients (median (25-75\%) 6.3 (4.4-7.3) $\mathrm{cm} v 4.7(2.2-7.1) \mathrm{cm}, \mathrm{p}=0.17)$.

Concerning the IBDQ scores, the younger HPN patients did significantly better (higher score) than older HPN patients with regard to systemic symptoms and social function, whereas no differences were seen with regard to bowel symptoms and emotional function. The overall IBDQ score tended to be better (higher score) in the younger HPN patients but the difference did not reach statistical significance $(p=0.076)$. An analysis of the responses to individual questions on bowel symptoms and emotional function did not disclose any significant differences. In questions on systemic symptoms, the older patients scored worse (lower score) with regard to a feeling of energy $(2.0 v 4.0, \mathrm{p}=0.03)$, whereas none of the differences in answers to the other questions reached statistical significance. Furthermore, none of the differences in responses to questions on social function reached statistical significance.

EFFECT OF THE PRESENCE OF A STOMA, DIAGNOSIS, AND DURATION OF HPN ON SIP AND IBDQ SCORES

\section{Presence of a stoma}

To evaluate the effect of the presence of a stoma on quality of life scores, both the non-HPN and HPN patients were divided into those with a stoma and those without. Figure $3 \mathrm{~A}, \mathrm{~B}$ gives a comparison of the SIP and IBDQ scores between the non-HPN patients with and without a stoma, and figure $3 \mathrm{C}, \mathrm{D}$ gives the comparison between the HPN patients with and without a stoma. The median age did not differ significantly between the non-HPN patients with and without a stoma (49 $v 50$ years, $p=0.95$ ), the number of men did not differ significantly (10 of $19 v 11$ of 17), and most of the patients in both groups had inflammatory bowel disease (13 of 19 and 17 of 17). None of the non-HPN patients suffered from intestinal dysmotility. In these non-HPN patients no significant differences were observed for the SIP and IBDQ scores in patients with and without a stoma. In the HPN patients the 

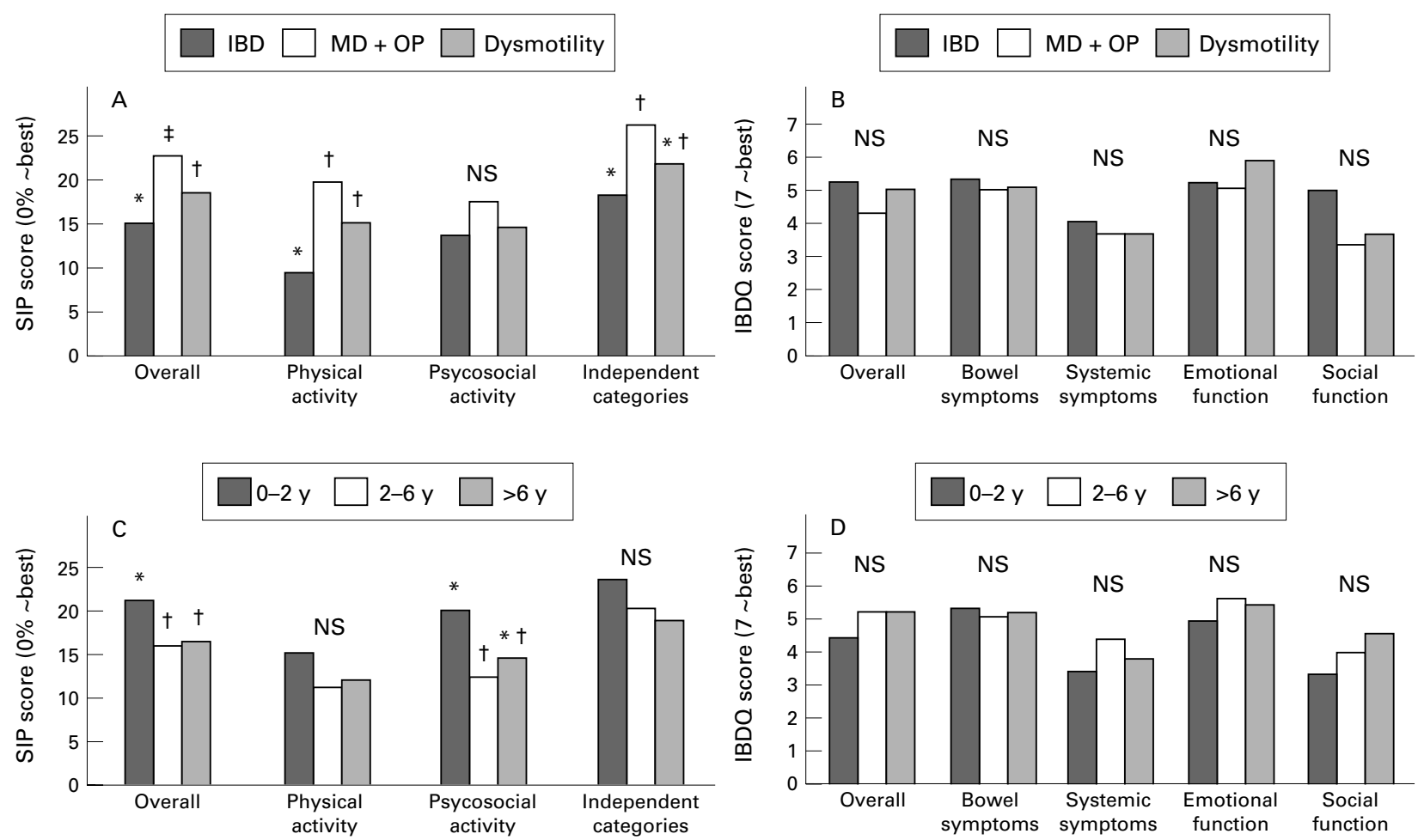

Figure 4 Mean sickness impact profile (SIP) scores and median inflammatory bowel disease questionnaire (IBDQ) scores according to diagnosis (A and $B)$ and duration of home parenteral nutrition (HPN) (C and D). IBD, patients with inflammatory bowel disease; MD+OP, patients with intestinal resection because of mesenteric vascular disease or complications of intra-abdominal surgery; dysmotility, patients with intestinal dysmotility. *, $\dagger$ and $\neq$ denote significant difference $(p<0.05)$ by the $\chi^{2}$ or Fisher exact test.

median age in the patients with a stoma was significantly higher than in those without $(49 v$ 32 years, $\mathrm{p}<0.01)$ and the number of men tended to be higher ( 6 of $11(55 \%) v 11$ of 38 $(29 \%))$. Furthermore, most (28 of 38$) \mathrm{HPN}$ patients with a stoma suffered from inflammatory bowel disease whereas most HPN patients without a stoma suffered from intestinal dysmotility (6 of 11). In these HPN patients without a stoma, a significantly better (lower) overall SIP score was observed, as well as in the physical and psychosocial dimensions but not the independent categories, compared with HPN patients with a stoma. Concerning the IBDQ scores, the HPN patients without a stoma only scored better (lower score) on emotional function. The non-HPN patients with a stoma were subsequently compared with the HPN patients with a stoma to evaluate the effect of HPN per se. The number of HPN and non-HPN patients without a stoma was too small for meaningful analysis. The faecal weight did not differ significantly in the non-HPN patients with a stoma compared with the HPN patients with a stoma (1.59 v $2.25 \mathrm{~kg} /$ day, $\mathrm{p}=0.21$ ). The HPN patients with a stoma scored significantly worse (higher score, fig 3C) compared with the non-HPN patients with a stoma (fig $3 \mathrm{~A}$ ) with regard to the overall SIP score $(18 \% v 7 \%, \mathrm{p}<0.001)$, the physical $(14 \% v 4 \%, \mathrm{p}<0.001)$ and psychosocial $(16 \%$ v $8 \%, \mathrm{p}<0.001)$ dimensions, and the independent category $(21 \% v 8 \%, \mathrm{p}<0.001)$. Concerning the IBDQ scores, the HPN patients with a stoma did significantly worse (lower score, fig 3D) than the non-HPN patients with a stoma (fig 3B) with regard to the overall IBDQ score $(4.9 v 5.8, \mathrm{p}<0.01)$, the bowel $(5.2 \vee 6.1, \mathrm{p}<0.05)$ and systemic symptoms $(3.7 \vee 5.4, \mathrm{p}<0.01)$, and the emotional function $(5.2 \vee 6.0, \mathrm{p}<0.05)$. The score for social function tended to be worse (lower score) $(3.9 v 4.8, \mathrm{p}=0.05)$.

\section{Diagnosis}

The HPN patients were divided into three groups according to diagnosis (fig $4 \mathrm{~A}, \mathrm{~B}$ ): one group of 31 patients ( 11 men), median age 44.8 years, had inflammatory bowel disease; another group of eight patients (two men), median age 58.1 years, had had an intestinal resection because of mesenteric disease or complications during surgery; the third group of 10 patients (four men), median age 48.4 years, suffered from intestinal dysmotility. The three groups differed significantly according to the overall SIP scores. The patients with intestinal resection due to mesenteric disease or complications during surgery had the worse scores (higher score) (23\%), the patients with dysmotility did slightly better (19\%), and the patients with inflammatory bowel disease had the best score (lower score) (15\%). In general, this pattern was observed in the physical and psychosocial dimensions and in the independent categories. No significant differences were observed for the IBDQ scores in the three groups according to diagnosis.

\section{Duration of HPN}

Figure 4C, D segregates the HPN patients according to duration of HPN. Eight patients (three men), median age 44.5 years, had received HPN for less than two years. Twenty 
three patients (nine men), median age 48.1 years, had received HPN for between two and six years, and 18 patients (four men), median age 45.1 years, had received HPN for more than six years. The patients who had received HPN for less than two years scored significantly worse (higher score, fig 4C) than those with a longer duration of HPN measured by the overall SIP score. A significant difference was not seen with regard to physical activity and independent categories, but patients who had received HPN for less than two years did worse (higher score, fig 4C) than the group of patients who had received HPN for two to six years. The differences in the IBDQ scores in the three groups did not reach statistical difference.

\section{Discussion}

This study evaluated the impact of HPN on quality of life in a total cohort of patients, representing $75 \%$ of all $\mathrm{HPN}$ patients, receiving HPN in Denmark. A non-disease specific behavioural measure of health status, the SIP, and a disease specific instrument, the IBDQ, were used and a group of non-HPN patients with either a functional (energy malabsorption $>2 \mathrm{MJ} /$ day) or an anatomical short bowel syndrome $(<200 \mathrm{~cm}$ of remnant small intestine) served as controls.

In the non-disease specific behavioural measurement by the SIP, the HPN patients scored worse (higher score) overall, in the dimensions and in the individual categories compared with non-HPN patients (fig 1). In the disease specific questionnaire, IBDQ, the HPN patients scored worse (lower score) overall, and in the four aspects of life, except for the bowel symptoms, compared with the nonHPN patients (table 2). One could argue that, as the presence of a stoma was more pronounced in the HPN patients than the non-HPN patients, this could be a major cause of the impairment of quality of life, as proposed previously. ${ }^{12}$ However, when the SIP and IBDQ scores in the sex and age matched nonHPN patients with and without a stoma were compared in this study, the presence of a stoma was not associated with poorer quality of life (fig 3). It therefore seems that the impairment in physical/systemic function, psychological/ emotional function, and social function in the HPN patients described by the two questionnaires may be related to the complex technology required for nutritional support rather than the inconveniences and bowel symptoms that accompany intestinal failure.

The HPN patients had reduced reserves of strength for physical activity, not only evidenced by the worse SIP scores (higher score) with regard to ambulation, mobility, and body care and movement, but also by reduced activity in connection with home management and a low rate of employment. Only $14 \%$ of the HPN patients were in full time work (fig 1), which corresponds to the employment rate of $20 \%$ in HPN patients in the United Kingdom given by Richards and Irving ${ }^{13}$ in a recent survey. The employment rate was, however, reported to be much higher $(52 \%)$ in a multi- centre survey in Europe, but about $20 \%$ of these patients had part-time work. ${ }^{14}$ The low employment rate in this study may be due to tiredness, abdominal pain, and diarrhoea reported by the HPN patients, but another explanation may also be found in the Danish National Health support and in illness related financial benefits. To reduce financial stress, all except seven HPN patients were granted a disability or retirement pension in Denmark. Five of the remaing seven patients were students. It is not possible to predict whether the pension influenced the patients' responses. The reduced reserves of strength for physical activity was most pronounced in women and HPN patients older than 45 (fig 2). A lower employment rate and more pronounced problems with respect to home management was seen in these patients.

The HPN patients expressed feelings of anger as a result of the bowel disease, which has also been described by Price and Levine. ${ }^{15}$ The HPN patients experienced loss of independence, social and work status, and control of bodily functions. The initial series of emotional reactions (disbelief, denial, hopelessness, pain, sorrow, fear, anger, and depression) may, however, be followed by an eagerness to learn the principles of their new life support system, supported by their family and the hospital staff. ${ }^{16}$ Acceptance of the new way of life may depend on personality, a stable marital relationship, and the level to which their physical health is restored. ${ }^{17}$ Emotionally the female HPN patients expressed more feelings of depression and discouragement in this study, and they appeared to be more tearful and upset than the male HPN patients (table 2). With the IBDQ no differences were encountered with regard to emotional function between the younger and older HPN patients, but the SIP questionnaire showed emotional stability to be poorer in the older patients.

Diminished sex drive has been recorded in chronically ill patients. ${ }^{15} 18$ In this study $43 \%$ of the HPN patients reported reduced sexual activity in the SIP questionnaire, and $55 \%$ of the HPN patients reported that their bowel problem had reduced their sexual activity in the IBDQ. This tendency was equal in both sexes and no differences were seen in the two age groups.

An effect of HPN on social interaction has also been shown by both questionnaires in this study (fig 1 and table 2). A difference could not be shown between sexes, but in the IBDQ the older HPN patients scored worse (lower score) than younger HPN patients, because of the tendency of a lower employment rate (fig 1). The HPN patients scored worse (higher score) than non-HPN patients with regard to recreation and pastimes in the SIP, but no impact of sex or age could be shown.

As the younger HPN patients have the best quality of life scores, it is interesting that these patients also are the most keen on a small bowel transplantation. ${ }^{19}$ This probably reflects the unwillingness to accept long term HPN. Rovera and colleagues ${ }^{20}$ investigated quality of life after intestinal transplantation and HPN. 
The nine intestinal transplant recipients reported a significant disruption in most areas of their lives when comparing "before illness" with "during illness" (while on HPN). When comparing "post-transplant" with "during illness" (while on HPN), they experienced a significant improvement in most areas of quality of life and they reported that quality of life after the transplantation compared favourably with their state before the illness. The study may, however, be subject to bias, as results were probably only obtained from the patients who had had a successful transplantation.

Although the results of small bowel transplantation look increasingly promising, the well adjusted adult patients on HPN are being advised to continue with HPN. However, when the survival after small bowel transplantation improves, and when measurements of quality of life in these patients compare well with life on HPN in a larger series, recommendations may change.

The quality of life in Danish patients with Crohn's disease and their healthy "buddy controls" was recently studied using the IBDQ. ${ }^{21}$ In the "buddy controls" the scores in the systemic, emotional, and social dimensions (median (25-75\%)) were 5.8 (5.2-6.4), 6.0 (5.7-6.5), and $7.0(6.2-7.0)$ respectively. In the patients with Crohn's disease the scores were significantly reduced to $5.2(4.2-5.9), 5.9$ (5.3-6.3), and 6.1 (5.5-7.0). This compares well with the non-HPN controls in this study, who scored 5.2 (3.9-5.9), 5.8 (5.4-6.4), and 4.8 (4.5-5.8) in systemic, emotional, and social dimensions. A further decline in the scores was seen in the HPN patients, who scored 3.8 $(2.8-5.4), 5.3(4.4-6.2)$, and $4.3(3.4-5.5)$ in the three dimensions.

Results obtained using the non-disease specific SIP questionnaire may be compared with those for patients with other chronic diseases. Laupacis and colleagues ${ }^{22}$ investigated the quality of life in a cohort of 168 patients with end stage renal disease treated by dialysis who were on a transplant waiting list. Mean age was 42 years, and 104 of the patients were men. The SIP scores for the total physical and psychosocial dimensions were $6.4 \%$ and $12.4 \%$ respectively compared with $13 \%$ and $14 \%$ in HPN patients in this study. The SIP scores for the independent categories sleep and rest, eating, work, home management, and recreation and pastimes were $24,9,40,16$, and $32 \%$ respectively compared with $21,20,86,25$, and $32 \%$ in HPN patients in this study. The overall SIP score was $13 \%$ in the patients with renal disease compared with $17 \%$ in HPN patients.
Thus the reduced quality of life compares with that reported in patients with chronic renal failure treated by dialysis.

Although HPN has evolved to be a successful, advanced, widespread, and lifesaving technique in the management of intestinal failure, the future quality control of medical care must also focus on the optimum rehabilitation of the HPN patient, not only with respect to physical function and survival, but also psychosocial and emotional function, aiming at the best possible overall quality of life in these patients.

1 Fleming CR, Remington M. Intestinal failure. In: Hill GL ed. Nutrition and the surgical patient. New York: Churchill Livingstone, 1981:219-35.

2 Grant D. Current results of intestinal transplantation. The International Intestinal Transplant Registry. Lancet 1996; Internationa

3 Abu-Elmagd K, Reyes J, Todo S, et al. Clinical intestinal transplantation: new perspectives and immunologic considerations. F Am Coll Surg 1998;186:512-27.

4 Detsky AS, McLaughlin JR, Abrams HB, et al. Quality of life of patients on long-term total parenteral nutrition at home. f Gen Intern Med 1986;1:26-33.

5 Bergner M, Bobbitt RA, Carter WB, et al. The sickness impact profile: development and final revision of a health status measure. Med Care 1981;19:787-805.

6 Guyatt G, Mitchell A, Irvine EJ, et al. A new measure of health status for clinical trials in inflammatory bowel disease. Gastroenterology 1989;96:804-10.

7 Temkin N, McLean A, Jr, Dikmen S, et al. Development and evaluation of modifications to the sickness impact profile for head injury. $\mathcal{F}$ Clin Epidemiol 1988;41:47-57.

8 Bergner L, Hallstrom AP, Bergner M, et al. Health status of survivors of cardiac arrest and of myocardial infarction controls. Am f Public Health 1985;75:1321-3.

9 Jeppesen PB, Staun M, Mortensen PB. Adult patients receiving home parenteral nutrition in Denmark from 1991 receiving home parenteral nutrition in Denmark from 1991 Scand F Gastroenterol 1998;33:839-46.

10 Cummings JH, James WP, Wiggins HS. Role of the colon in ileal-resection diarrhoea. Lancet 1973;i:344-7.

11 Schofield WN. Predicting basal metabolic rate, new standards and review of previous work. Hum Nutr Clin Nutr 1985;39(suppl 1):5-41.

2 Nightingale JM. The Sir David Cuthbertson Medal Lecture. Clinical problems of short bowel and their treatment. Proc Nutr Soc 1994;53:373-91.

13 Richards DM, Irving MH. Assessing the quality of life of patients with intestinal failure on home parenteral nutrition. Gut 1997;40:218-22.

14 Messing B, Landais P, Goldfarb B, et al. Home parenteral nutrition in adults: a multicentre survey in Europe. Clin Nutr 1989;8:3-9.

15 Price BS, Levine EL. Permanent total parenteral nutrition: psychological and social responses of the early stages. 尹PEN F Parenter Enteral Nutr 1979;3:48-52.

16 Robinovitch AE. Home total parenteral nutrition: a psychosocial viewpoint. FPEN 7 Parenter Enteral Nutr 1981;5:5225.

17 Ladefoged K. Quality of life in patients on permanent home parenteral nutrition. FPEN F Parenter Enteral Nutr 1981;5: $132-7$.

18 Levy NB, Wynbrandt GD. The quality of life on maintenance haemodialysis. Lancet 1975;i:1328-30.

9 Carlson GL, Maguire G, Williams N, et al. Quality of life on home parenteral nutrition and attitudes towards intestinal transplantation. A single centre study of 37 patients. Clin Nutr (in press).

20 Rovera GM, DiMartini A, Graham TO, et al. Quality of life after intestinal transplantation and home parenteral nutrition [abstract]. 5th International Symposium on Intestinal Transplantation 1997;1:23-O16.

21 Kruuse C, Guassora AD, Thomsen OØ, et al. Subjective Kruuse C, Guassora AD, Thomsen OØ, et al. Subjective
health status and social function in patients with Crohn's disease [abstract]. Gastroenterology 1996;110:A450

22 Laupacis A, Keown P, Pus N, et al. A study of the quality of life and cost-utility of renal transplantation. Kidney Int 1996;50:235-42. 